\title{
La figura del pishtaco andino como expresión simbólica de trauma social, aculturación y conflicto (ss. XVI-XXI)
}

\author{
Mirosław Mąka, Elżbieta Jodłowska
}

\begin{abstract}
Resumen
El pishtaco andino es una de las figuras más intrigantes y difíciles de definir dentro del imaginario popular. No cabe en ninguna de las categorías de seres sobrenaturales presentes en el universo de las creencias de los pueblos andinos. Crea su categoría propia, autónoma, que genéticamente radica tanto en la realidad histórica de las primeras décadas de la conquista, como en la mitología autóctona. Constituye una conceptualización y visualización de la trauma cultural muy profunda y fruto de su tratamiento sicológico que ha atribuido una cara y una figura a las amenazas existenciales nuevas. El temor ante lo desconocido implicaría un estado de desamparo $y$, en cambio, el temor ante un enemigo conocido permite elaborar una estrategia de defensa. En la mitología milenarista andina del periodo siguiente a la Conquista el pishtaco apareció como una figura simbólica personalizando al enemigo que va a desaparecer solo cuando se reaviva el imperio Inca idealizado. El pishtaco no viene dotado de una sola cara o un solo aspecto. En los últimos 500 años aparecía llevando vestido de convento, del hacendado cruel, de un soldado, de un médico o cualquiera otro a quien los campesinos hubiesen reconocido como "ajeno". Las visualizaciones más antiguas que se han descrito ya en el siglo XVI siguen siendo vivas y le suceden, sobreponiéndose a ellas, las visualizaciones siguientes, creadas más tarde. Es sorprendentemente viva la creencia en la existencia del pishtaco hasta en los tiempos contemporáneos. Esto da fe de una estructura interna perfecta de la misma que ha sido capaz de adaptarse a unas formas de amenaza contemporáneas. El pishtaco ataca a los indios, les degüella y extrae su grasa que no consume (;no es un variante de vampiro!) sino la transfiere a manos de los blancos en donde se utiliza para resforzar los equipos que fabrican ellos. Los relatos más antiguos y más populares en Perú cuentan como se añadía la grasa de los indios a las aleaciones de metales, a ordenadores o teléfonos. La lógica de esta creencia viene apoyada sobre una valorización extraordinaria de la grasa humana en las sociedades andinas tradicionales quienes veían en ella una sustancia y un poder vitales. El pishtaco mata al indio y le extrae su grasa para que su fuerza vital sirviese al enemigo. Esto incluye una simbólica universal y una metáfora del conflicto eterno entre los pueblos dominados de los Andes y las diferentes impersonaciones de sus enemigos. El pishtaco es una figura del temor ante la amenaza a su propia existencia física y una aculturación que lleva hacia la
\end{abstract}

\footnotetext{
Mirosław Mąka • Instituto de Etnología y Antropología de la Cultura de la Universidad Jaguelónica, fallecío el 14 de noviembre de 2020

Elżbieta Jodłowska - Instituto de Etnología y Antropología de la Cultura de la Universidad Jaguelónica, ela@ejm.pl
} 
destrucción de la identidad conjunta de este grupo social. Paradójicamente, sin embargo, se ha vuelto un factor integrante de los que creen en ella identificando al enemigo y movilizándoles a defenderse.

Palabras clave: Andes, pishtaco, visualización del enemigo, grasa humana, trauma colectiva, conflicto cultural

\section{ANDEAN PISHTACO AS A SYMBOLIZATION OF SOCIAL TRAUMA, ACCULTURATION AND CONFLICT (XVI-XXI C.) \\ Abstract}

The Andean pishtaco is one of the most intriguing and difficult to define figures in the collective imagination. It does not fit into any of the categories of supernatural beings present in the beliefs of the Andean peoples. It exists within its own, autonomous category, genetically rooted in the historical reality of the first decades of the conquistadors, as well as in the indigenous mythology. It is a conceptualization and visualization of a deep cultural trauma and its psychological resolution, which provides imaging for the new, unknown existential fears. Fear of the unknown establishes the emotion helplessness, while fear of a known enemy enables development of defense strategies. In Andean millenarian myths of the period after the Conquista the pishtaco came into existence as a symbolic figure of an enemy which would disappear only after the awaited revitalization of the idealized Incan empire. Pishtaco has neither one single face, or even one single appearance. During the last 500 years it appeared in the costumes of a monk, a cruel Spanish hacendado, a soldier, a doctor, or anyone perceived as a "stranger" by traditional campesinos. The oldest visualizations, documented as early as in the XVI c., are still alive today, interconnected with the ones created later. Even today the belief in the pishtaco remains surprisingly strong. It constitutes a proof for a perfect internal structure of this belief, allowing it to adapt to the new threats. Pishtaco attacks the Indians, kills them and melts their fat, however, it does not eat it (since it is not a variation of a vampire!), but transfers it to the White Men, who use it to harden the devices they produce. The oldest and most common oral lore tells about adding the Indian fat to the metal alloy used to create bells. The newer lore tells about using this fat to power vehicles, computers, or telephones. The logic of this belief is based on an extremely positive valorization of fat in the traditional Andean communities, that have seen it as a substance of life and vital powers, since the earliest times. Pishtaco kills Indians and takes away their fat in order, and in this way their vital power serves the enemy. This belief contains universal symbolism and the metaphor of eternal conflict between the dominated Andean peoples, and different embodiments of their enemies. Pishtaco is a figure of fear of a threat to the physical existence, and of acculturation that leads to the destruction of the group identity. Ironically, it is also an integrating aspect for those, who believe in it, allowing to identify the enemy, and mobilizing to defense.

Keywords: Stowa: Andes, pishtaco, enemy visualization, human fat, collective trauma, cultural conflict

Es intrínseco de la mitología inca - heredera de numerosas tradiciones más antiguas - el concepto de lo cíclico de las épocas, separadas las unas de las otras por las sucesivas crisis causadas por eventos de dimensiones apocalípticas. Cada una de esas épocas tenía su propia secuencia temporal componiendo al mismo tiempo conjuntamente un caudal de sucesos consecutivos de carácter lineal - un sui generis ,tiempo universal” naciendo del caos original, antecedente de la cosmogonía y que no tiene, en cambio, según la narración del mito, ningún fin. Las crisis apocalípticas pueden percibirse como giros de los sistemas que han perdido su dinámica interna padeciendo en lo sucesivo una atrofia. El concepto de pacha kuti panandino define su naturaleza que incluye tanto un factor de su destrucción como uno de su renacimiento en una forma nueva, distinta. Sobre los escombros de las formas de organización antiguas van naciendo unas nuevas, dirigiendo la lógica de su suce- 
sión el principio fácil de percibir que es él del perfeccionamiento de civilizaciones. La mitología andina describe las épocas que se sucedían desde la creación del mundo y la aparición de los „Hombres Piedra Viva”(Wari Wira Qucha Runa y Wari Runa) pasando por el periodo de la barbarie (Purum Runa), él de hombres guerreros (Awqua Runa) terminándose en el periodo de los hombres Incas (Inqap Runan o bien Inqa Pacha Runa) (Szemiński 1983; Szemiński, Ziółkowski, 2014).

El corriente de la mitología milenarista que se formó ya en la fase temprana de la Conquista incluye a un periodo nuevo dentro de los ciclos de los tiempos, trágico para los indios, que es él de la conquista, considerándose como otra secuencia apocalíptica o pacha kuti más. Dentro de la misma se da un „reverso” total del mundo cuyo efecto es el cambio de poder sin precedentes y que iba en contra de su tradición apareciendo junto con la amenaza hacia las bases de su existencia y de su cultura. El cataclismo de la conquista que se iba prolongando y abarcando las generaciones siguientes no deja de ser un pacha kuti. Todo lo contrario - va cobrando gradualmente los rasgos de una época autonómica en su historia. Una época, por cierto, muy particular - privada de continuidad respecto a las épocas anteriores y discontinuando el principio del perfeccionamiento de su civilización. Es todo lo contrario - desde la perspectiva de los Indios se trata de una época degradante y barbarizante respecto al esplendor de la civilización de Tawantin Suyu (Szemiński 1992).

En la época de terror, explotación y aculturación no desaparece la idea de la continuidad de los tiempos, encontrando su expresión en la previsión milenarista de una reactivación del Imperio Inca idealizado. Pasando los siglos y hasta los tiempos contemporáneos se van repitiendo en los Andes los relatos de una cabeza de Ataw Wallpa escondida que va reactivándose, adquiriendo cada vez más partes del cuerpo de su gobernante (Arguedas 1981, 175-179). La relación mítica de su persona con el organismo teístico del estado tiene carácter de una identificación ontológica que sale más allá de la metáfora. La reavivación del Inca supone al mismo tiempo una reconstrucción del Tawantin Suyu - conceptualmente posible solo en combinación simétrica con el renacimiento biológico de su gobernante. Tanto el proceso biológico como el histórico explicita el mismo sentido en la unidad homogénea del cosmos andino.

Observamos pues el proceso de establecerse dos capas de interpretación del periodo histórico corriente complementarias: por un lado una apocalipsis pacha kuti distribuida en el tiempo y, por el otro, un proceso de creación de una época nueva que viene escondido en forma de una copia del mundo de antes de la conquista utópica e idealizada. Este mito tiene para nosostros una importancia particular ya que reaparece en la misma un actor clave del teatro de los sucesos andinos que ocurrieron en los últimos 500 años - la figura intrigante y sin precedentes del degollador llamado pistaco.

Dicha figura crece logrando las dimensiones de una metáfora de lo sucedido en esta parte del mundo, conformando el contenido del mito milenarista 
más importante del Perú de los últimos 500 años en el que juega un papel de alta importancia simbólica. Narra este mito que los pishtacos aparecieron a la par de la apocalipsis de la conquista y desaparecerán cuando ésta termine. La figura del degollador forma un paréntesis histórico en el que cabe el inicio y el fin del „mundo al revés" predicho. Vive en el mismo como una forma de ser nueva que densifica y concentra en sí los rasgos de relaciones culturales significativos que corresponden a la realidad traumática de una conquista brutal. El pishtaco es resultado de la transformación de esta realidad en categorías del lenguaje simbólico - es una creación del imaginario conjunto única que nació efecto de la interacción de factores históricos y sico-sociológicos profundamente modelados por los elementos de la tradición autóctona.

Es el momento para esbozar la figura modelo del pishtaco que lleva siglos persiguiendo a los indios andinos y siendo su propia pesadilla. La figura de un ser que despierta temor, despertando en no pocas ocasiones una psicosis del temor en comunidades que abarcan a aldeas y burgos enteros. Es un asesino de los indios, pero no se trata de un asesino en forma y significado común que supone un hombre que puede volverse uno que mata en diferentes circunstancias y con intensidad o grado variable. El pishtaco-asesino no es una aberración de la norma sino que lo asesino constituye la misma quinta esencia de su existencia. Es un ,asesino nato" en forma pura o sea un ser cuyo único condicionante y sentido de la vida es asesinar a los indios para extraerles o succionar grasa de sus cuerpos. Es importante en este lugar que hagamos una paréntesis que corrija los esquemas de pensar tanto populares como científicos: el pishtaco no es una variante de vampiro andina - estructuralmente su ser no tiene nada que ver con el vampirismo. No consume la grasa que ha extraído de los indios sino la transmite desde el espacio del ecúmeno indio al universo de los blancos y mestizos. Es solo un medio - un emisario de los que demandan esa grasa y, al mismo tiempo, su incorporación visual de los que le han despachado en esta misión. La grasa se va a las ciudades de la Costa del Pacífico, a sus iglesias y conventos, a las minas, fábricas y empresas. De allí se transfiere más lejos - a España y a los Estados Unidos o bien la aprovecha el gobierno de Perú en sus transacciones internacionales. Va alimentando todo género de equipos técnicos y vehículos que fabrican los blancos y es un componente precioso de sus cosméticos y fármacos. En la creencia popular de los habitantes de los Andes viva hasta hoy en día la forma de aprovecharlo más antigua ha sido la de añadirla a las aleaciones de metales que sirven a la fundición de las campanas. Los indios creen que el sonido de todas las campanas antiguas en Perú va reforzado y refinado por la grasa de los indios que contienen (Morote Best 1951-1952; Kapsoli 1991).

El aspecto del pishtaco descodifica su naturaleza. La visualización más antigua de esta figura es la de un hombre blanco, alto, barbudo con cara repulsante, vestido de hábito de convento provisto de una chapuza. Se han dado pishtacos vestidos de uniforme militar y en las últimas décadas unos pishtaco vistiendo terrucos 
propios a los miembros del Sendero Luminoso. En la parte boreal del Perú andino se incorporan en las figuras de hacendados crueles cabalgando - unas figuras recordadas hasta hoy en día por las generaciones de los indios más mayores. Las caras de algunos de ellos llevan abscesos y han subido deformaciones que les vuelven más feos características de las enfermedades que los españoles trajeron a Nuevo Mundo. La enfermedad es al mismo tiempo una figura personificando el mal a la que el imaginario popular da una forma concreta. El pishtaco suele ser un hombre blanco y, menos corrientemente, un mestizo. El catálogo de sus personificaciones no se limita a las que acabamos de enumerar - sigue abierto a unas nuevas que sin eliminar a las anteriores no dejan de hacer crecer su número. El fenómeno del pishtaco consiste en el que su figura no queda encerrada en la esfera de la demonología o leyendas del pasado sino que existe en la continuidad homogénica de la tradición y de lo contemporáneo constituyendo tanto un elemento del folklore popular como parte de la realidad cotidiana. Puede tomarse por pishtaco cualquier "ajeno" a los indios runa llevando una vida tradicional si éstos últimos encuentran en tal indivíduo rasgos de un „enemigo” (Mróz 1992). Las diferentes visualizaciones del pishtaco devuelven la imagen de las diferentes formas de „enemistad” y las de un mal absoluto. Esos seres ocupan un lugar intermedio entre la demonología y la realidad de un ser humano. Un pishtaco tiene rasgos superhumanos, permaneciendo al mismo tiempo un ser humano. Los indios son capaces de matarlo y muchas veces lo hacen estando convencidos que se trata de un acto de autodefensa. No escasean los casos de asesinatos de gringos blancos que se han tomado por pishtaco dando fe de lo vivo que sigue siendo esta creencia aunque pasa también que se aproveche para esconder los motivos de robo de los mismos (Jodłowska, Mąka 2016).

El fenómeno de la creencia en los pishtaco nos inclina a analizar con profundidad las razones de su perseverancia excepcional que resiste a los impulsos del desarrollo civilizatorio. Su explicación se esconde en la misma estructura de su figura y su lugar en e sistema de las visión del mundo tradicional andino que ha superado conceptualmente el choque cultural causado por la conquista creando simbolizaciones adecuadas a la nueva realidad. La figura del pishtaco que se formó en espacio de apenas unas decenas de años tras la llegada de los españoles se volvió un elemento de las creencias andinas permanente hasta nuestros tiempos sin que aparezca tendencia alguna de su desaparición a pesar de que se haya separado una corriente comercial de sus mutaciones deformadas dentro de la cultura pop. El polisemantismo contemporáneo del nombre del pishtaco que aparece sobre todo en los sociolectos y en el habla cotidiano junto con la ampliación de las zonas geográficas y culturales en las que aparece esta figura no parecen debilitar su vivacidad en las zonas andinas (Taylor 1991; Taussig 1980). Puede ser su explicación la continuidad histórica de las razones de su ser indispensables para que siga siendo estructuralmente estable. Dichas condicionantes o sea las fuentes de temor, frustraciones, experiencias traumáticas y amenazas reales o anticipadas que vienen del „mundo exterior” de los conjuntos de 
la población cumplen con el mismo papel dentro del sistema idiosincrático de los indios quedando el pishtaco una construcción perfecta visualizando las diferentes formas de enemistad en el lenguaje de simbolización visual. Las formas de tensión y conflicto nuevas crean una visualización adecuada de esta figura denotando la imagen del „enemigo” y una tela de fondo de relaciones antagonistas (Zapata Gastón 1984). La trauma cultural profunda que supuso de la conquista y que dio luz a la figura del pishtaco continuó en forma de las muchas guerras, agresiones desde el exterior, explotación, penas y casos de exclusión social que los indios iban experimentando a lo largo de los siglos de la mano de los blancos, mestizos y otros ,ajeno"” (Nowicka 1972). El conflicto cultural multigeneracional creó barreras de desconfianza y separación inamovibles siendo estas medios de defensa de su propia existencia e identidad. La fijación de dichas barreras con carácter de prejuicios y estereotipos iba apoyado por un factor subyacente, biológico - la herencia epigenética de la trauma a través de muchas generaciones (Hałas 2013).

En la actualidad no han desaparecido, ni mucho menos, las fuentes serias de conflictos. Las investigaciones que llevamos a cabo en 2016 en el departamento de la sierra de Ancash demostraron un antagonismo creciente de los indios para con los ajenos, sobre todo hacia los consorcios mineros americanos y japoneses que iba a par con un sentimiento de ser explotados de forma rapaz por ellos. Una consciencia ecológica cada vez más popular va fortaleciendo tales opiniones lo que, a su vez, se propagan al crecer el número de las minas y al degradarse la naturaleza de esta parte de los Andes. Al mismo tiempo - lo que puede sorprender - este fenómeno viene acompañado por la convicción de que la actividad de pishtaco va aumentando y cada vez son más numerosos los casos de desaparición de personas vista la demanda creciente de la grasa de los indios, indispensable para mantener los equipos mineros. El valle del Rio Santa es solo uno de los muchos focos de conflicto adormecidos pero vivos en los Andes en donde el pishtaco activa su procedimiento asesino (Jodłowska, Mąka 2016, 204-212).

El pishtaco como un „medio” vistiendo los vestidos del „enemigo” de los indios constituye solo su característica de forma parcial. Los relatos desvelan un catálogo largo y homogéneo de sus rasgos, características y normas opuestas a las autovisualizaciones que crean de sí los mismos indios definiendo la identidad de su grupo. El pishtaco tiene cada vez otro aspecto diferente del indio runa, no habla quechua, no mastica las hoja de la coca, no bebe chicha, puede estar enfermo o propagador de enfermedades, es estéril o sexualmente desviado, manejo atributos de culturas ajenas (p.ej. un libro) moviéndose en el espacio orbis exterior - un espacio temporal opuesto al del orbis interior indio. Es habitante de un mundo à rebours agresivo que amenaza la existencia y la identidad cultural de los habitantes de los Andes.

Constituye un elemento indispensable y, al mismo tiempo, clave que explica el sentido de la creencia en el pishtaco descodificando por completo su lógica 
mítica la grasa de los indios. El mito del pishtaco requiere una respuesta a la pregunta elementar - ¿por qué esta sustancia precisamente es el objeto de la demanda de los „enemigos” de los indios? La respuesta no la vamos a encontrar sin embargo del lado de aquellos „enemigos" sino en la misma cultura que ha creado este mito. Nos acercaremos a ella si sacamos conclusiones de las connotaciones específicas que tiene en los Andes la grasa humana. Sin conocerlas no vamos a entender es estatus particular del que goza esta sustancia como objetivo de la demanda asesina del pishtaco. No vamos a poder entender tampoco el sentido de la existencia del pishtaco mismo ni interpretar de forma correcta la motivación del uso de la grasa de los indios como componente de campanas, máquinas, vehículos y fármacos.

Cabe, sin embargo, rechazar de forma contundente la interpretación que sigue siendo aplicada en los trabajos que tratan el tema del pishtaco que quiere que la grasa de los indios se trate en términos racionales y materialistas como si perteneciese a la categoría de „materia prima”, „componente” o bien „lubricante”. Su sentido real es mucho más profundo y tiene que ver con la percepción extremadamente afirmativa de la grasa humana la cual se ha formado en las culturas andinas desde hace miles de años. Ya en el nombre del dios-creador más antiguo Wira Qucha, la partícula „Wira” significa „grasa” y al mismo tiempo „propagador de la vida” o ,agua que da vida” (Szemiński, Ziółkowski 2014). En los textos mitológicos incas y en las súplicas de rezamientos la grasa humana aparece directamente o de forma metonímica como un sustituto del semen. Es entonces una sustancia vital concentrada la cual queda, de por su naturaleza, marcada con el rasgo de inmortalidad. Es un componente procreativo del cuerpo humano que condiciona la transmisión de la vida de generación a generación. El pishtaco al asesinar a un indio y extrayendo su grasa está matando al mismo tiempo a sus sucesores potenciales y, en un plano más amplio, corta las bases de la supervivencia de toda una sociedad. Un asesinato no es lo que parece ser, es un asesinato multiplicado ad inifnitum - un asesinato que es al mismo tiempo real y simbólico. En este contexto cabe recordar lo importante que ha sido el establecer que el pishtaco asesinaba solo a hombres en edad procreativa. El análisis que llevamos a cabo de las numerosas leyendas y cuentos sobre el pishtaco ha demostrado que los que proporcionaban la grasa no han sido nunca niños, mujeres o ancianos sino precisamente hombres en edad procreativa. No se ha detectado esta particularidad en la literatura científica que tarta el tema del pishtaco hasta la fecha y, sin embargo, es absolutamente clave. Constituye el tramo indispensable que completa el paradigma sin el que queda incomprensible. La grasa humana considerada como un elemento vital se vuelve un signo claro y un elemento clave del comunicado que describe esa relación intercultural antagonista. El final de esta relación y, al mismo tiempo, la confirmación de la afiliación que tiene la grasa como elemento fertilizante-vital es su ,vida” post-mortem - fuente de energía inagotable que refuerza el sonido de campanas, es fuerza motriz de las máquinas y concede el poder de curar a fármacos. Estas convicciones tienen que ver 
igualmente con la percepción arcaica, personificante de objetos ajenos que se mueven y emiten sonidos como seres reales que tienen vida (Sullivan 2007). Un indio y, sobreentendido - también su nación -muere pero la grasa que le ha sido extraída, marcada por inmortalidad - sigue sirviendo a su agresor y „enemigo” quedando explotada en su universo. Sería difícil encontrar una visualización más expresiva que resuma mejor la esencia del conflicto intercultural extremadamente fuerte desde el punto de vista de una víctima potencial. La estructura del mito del pishtaco refleja el paradigma de todos los conflictos, amenazas, agresiones y dominación sobre los indios posibles. Es un modelo universal creado sobre los baluartes de la defensa de su cultura - indispensable para la transcripción de un mal „desconocido" hacia unas nociones, categorizaciones y visualizaciones conocidas. Permite reconocerlo y expresarlo en el lenguaje de mito, atribuirle una figura, una cara y un nombre esto les lleva a poder digerir en el contexto cultural la crisis que están viviendo y encontrar una estrategia defensiva.

Los conflictos interculturales dan producen unos eventos históricos medibles y espectaculares pero al mismo tiempo generan fenómenos subyacentes de „larga duración” que se dan en la esfera de la interpretación mítica de la realidad. Mario Vargas Llosa - el único escritor de gran importancia que percibió la gran importancia cultural del fenómeno del pishtaco en su obra Lituma en los Andes guiado por su intuición etnológica ha resumido sus reflexiones en una síntesis simple y al mismo tiempo perfecta: „Cierto, éstos vienen siempre en los tiempos difíciles ” (Vargas Llosa 1993, 156; Wolfenzon 2011).

\section{Bibliografia:}

Ansión, Juan y Sifuentes, Eudosio

1984 La imagen popular de la violencia, a través de los relatos de degolladores, [en:] J. Ansión (ed.), Pishtacos de verdugos a sacaojos, Tarea, Lima, 61-108.

Arguedas, José María,

1981 Formación de una cultura nacional indoamericana, 197 pp., Siglo Veintiuno Editores, SA, Mexico-Madrid-Bogota.

Canessa, Andrew

$2000 \quad$ Fear and Loathing on the Kharisiri Trail: Alterity and Identity in the Andes, „The Journal of the Royal Anthropological Institute” Vol. 6, $\mathrm{N}^{\circ}$ $4,705-720$.

\section{Fabulaciones...}

1989

...de tres mundos. Mitos, leyendas, tradiciones y cuentos folclóricos peruanos, 285 pp., recopilación y selección de Carmen Sebastiani, Ediciones Pedernal, Lima. 
Hałas, Elżbieta

2013 Pamięć podzielana i pamięć podzielona: symbolizacja i neutralizacja traumy, [en:] Barbara Bossak-Herbst, Małgorzata Głowacka-Grajper, Michał Kowalski (eds.), Antropologiczne inspiracje. Księga Jubileuszowa dla Profesor Ewy Nowickiej, Wydawnictwa Uniwersytetu Warszawskiego, Warszawa, 324-331.

Herrera, Alexander y Lane, Kevin

2006. „¿Qué hacen aqui esos pishtaku?”: sueños, ofrendas y la construcción del pasado, „Antípoda” № 2 (enero-junio), 157-177.

Jodłowska, Elżbieta y Mąka Mirosław

2016 Pishtaco. Fenomen symbolizacji traumy kulturowej w spolecznościach Kapsoli, Wilfredo andyjskich, 243 pp., Zakład Wydawniczy „NOMOS”, Kraków.

1991 Los pishtacos: degolladores degollados, „Bulletin de Institut Français d'Etudies Andines", Vol. 20, N 1, 61-77.

Lopez Albujar, Enrique

1984 Nuevos cuentos andinos, Libreria „Studium”, Lima (capítulo: Huayna - Pishtanag, 35-69).

Marzal, Manuel M.[aría]

1983 La transformación religiosa peruana, 458 pp., Pontificia Universidad Católica del Perú, Lima.

Molinié Fioravanti, A.[ntoinette]

1991 Sebo bueno, Indio muerto: la estructura de una creencia andina, ,Bu1letin de Institut Français d'Etudies Andines" Vol. 20, № 1, 79-92.

Morote Best, Efraín

1998 El degollador: historia de un libro desafortunado, 112 pp. Universidad Nacional de San Cristóbal de Huamanga, Ayacucho.

Morote Best, Efraín

1951-1952 El Degollador. „Tradición, Revista Peruana de Cultura”, Año I2, Vol. 4 (septiembre 1951 - enero 1952), $\mathrm{N}^{\circ} 11,133-156$.

Mróz, Marcin

1992 Los runa y los wiraquča. La ideología social andina en la tradición oral quechua, 216 pp., Universidad de Varsovia, CESLA, Varsovia.

Nowicka, Ewa

1972 Bunti ucieczka. Zderzenie kultur i ruchy spoleczne, 286 pp., Państwowe Wydawnictwo Naukowe, Warszawa.

Oliver-Smith, Anthony

1969 The Pishtaco. Institutionalized Fear in Highland Peru, „The Journal of American Folklore" Vol. 82, N 326 (Oct.-Dec.), 363-368.

Posern-Zieliński, Aleksander

1974 Ruchy spoleczne i religijne Indian hiszpańskiej Ameryki Poludniowej 
(XVI-XX w.), 229 pp., Zakład Narodowy im. Ossolińskich, Wrocław.

Pribyl, Rosario de

2010 Evidencias médico antropológicas sobre origen del pishtaco, ,Revista Peruana de Medicina Experimental y Salud Pública” Vol. 27, $\mathrm{N}^{\circ} 1$, 123-137.

Riviera Andía, Juan Javier

2006 Mitología en los Andes, [en:] Alejandro Ortiz Rescaniere (ed.), Mitologias amerindias, Trotta, Madrid, 129-176.

Rivière, Gilles

1991 Lik'ichiri y kharisiri... A propósito de las representaciones del „otro” en la sociedad Aymara, „Bulletin de Institut Français d'Etudies Andines" Vol. 20, No 1, 23-40.

Salazar-Soler, Carmen

1991 El pishtacu entre los campesinos y los mineros de Huancavelica, „Bulletin de Institut Français d'Etudies Andines” Vol. 20, N 1, 7-22.

Spetale Hernández, Rubén

2015 Antología de mitos, leyendas y relatos ancashinos, Killa Editorial, Huaráz.

Sullivan, Lawrence E.

2007 Człowiek $i$ społeczeństwo jako kompozycje muzyczne, [en:] Katarzyna Zajda (ed.), Estetyka Indian Ameryki Poludniowej. Antologia, Univer-

Szemiński, Jan sitas, Kraków, 117-127.

1984a Myślenie po kiczua, „Etnografia Polska” T. 28, º. 2, 51-109.

Szemiński, Jan

1984b El único español bueno es el español muerto: maten a los españoles, [en:] Juan Ansión (ed.), Pishtacos de verdugos a sacaojos, Tarea, Lima, 19-60.

Szemiński, Jan

1992 El mundo andino dominado por los ,, muertos rebeldes”, [en:] Miguel León-Portilla (ed.), De palabra y obra en el Nuevo Mundo. 1. Imagines interétnicas, Siglo Veintiuno Editores, Mexico-Madrid-Bogota, 171-193.

Szemiński, Jan

1987 Las generaciones del mundo según den Felipe Guaman Poma de Ayala, „Historica” Vol. 7, № 1 (julio), 69-109.

Szemiński, Jan y Ziólkowski, Mariusz

2014 Mity, rytualy i polityka Inków, 472 pp., Państwowy Instytut Wydawniczy, Warszawa.

Taussig, Michael T.

1980 The Devil and Commodity Fetishism in South America, 295 pp., The 
University of North Carolina Press, Chapel Hill.

Taylor, Gerald

1991 Comentarios etnolinguísticos sobre el término pishtaco, „Bulletin de Institut Français d'Etudies Andines" Vol. 20, N 1, 3-6.

Toro Montalvo, César

1990-1991. Mitos y leyendas del Perú, 3 tomos, 1602 pp., AFA Editores Importadores S.A., Lima.

Vargas Llosa, Mario

1993 Lituma en los Andes, 262 pp., Editorial Planeta S.A., Barcelona.

Vergara Figueroa, Abilio y Ferrúa Carrasco, Freddy

1984 Ayacucho: de nuevo los degolladores, [en:] Juan Anción (ed.), Pishtacos de verdugos a sacaojos, Tarea, Lima, 123-136.

Weismantel, Mary

2001 Cholas and Pishtacos: Stories of Race and Sex in the Andes, with a Foreword by Catharine R. Simpson, 325 pp., The University of Chicago Press, Chicago and London

Wolfenzon, Carolyn

2011 El „Pishtaco” y el conflicto entre la costa y la sierra en „Lituma en los Andes” y „Madeinusa”, „Latin American Literary Reviev” Vol. 38, $\mathrm{N}^{\circ} 75$ (Jan.-June), 24-45.

Zapata Gastón, Antonio

1984 Sobre ojos y pishtacos [en:] Juan Ansión (ed.), Pishtacos de verdugos a saca-ojos, Tarea, Lima, 137-140. 